\title{
An in vitro Comparison of Root Canal Measurement in Permanent Teeth by Electronic Apex Locator, Conventional and Digital Radiography
}

\author{
${ }^{1}$ Musab Hamed Saeed, ${ }^{2}$ Alexander MJ Luke, ${ }^{3}$ Nazil A Abtahl, ${ }^{4}$ Praveen Pradeep A \\ ${ }^{1}$ Assistant Professor, Department of Restorative Dentistry, Ajman University of Science and Technology, Ajman, UAE \\ ${ }^{2}$ Oral and Maxillofacial Radiologist, Department of Oral Medicine and Radiology, Ajman University of Science and Technology, Ajman, UAE \\ ${ }^{3}$ Clinical Tutor, Department of Restorative Dentistry, Ajman University of Science and Technology, Ajman, UAE \\ ${ }^{4}$ Senior Research Fellow, All India Institute of Medical Sciences, New Delhi, India
}

Correspondence: Alexander MJ Luke, Oral and Maxillofacial Radiologist, Department of Oral Medicine and Radiology, Ajman University of Science and Technology, Ajman, UAE, e-mail: alexvinod@gmail.com

\section{ABSTRACT}

Introduction: In endodontic treatment for permanent teeth, it is important to estimate the working length precisely. Currently, various methods are used in clinical practice for working length estimation. The objective of this study is to compare the accuracy of root canal length in permanent teeth determined by electronic apex locators, conventional and digital radiography.

Materials and methods: A total of 50 single rooted permanent teeth with mature apices were extracted for periodontal reasons and obtained from different sources without caries were studied. The radiographic measurements were done using paralleling technique. Electronic measurements were done next using third generation, DentAport ZX. Actual canal length of each tooth was measured using direct observation of the apical exit of the file. Measurements obtained from radiographic and electronic methods were compared to the actual tooth length. Interclass correlation coefficients and failure rate for each method and their combinations were calculated using STATA 12.0 software. Results: The measurements obtained through all the three techniques showed high interclass correlation coefficients and excellent level of agreement. The failure rate of measurements obtained through conventional radiography, digital radiography and electronic apex locators was 38,30 and $22 \%$ respectively. Combination of conventional radiography with electronic apex locator showed an accuracy of $90 \%$, while combination of digital radiographs and electronic apex locators given an accuracy of $96 \%$.

Conclusion: A combination of digital radiographic and apex locator methods for determining the root canal length in permanent teeth can be considered safe, reliable and precise.

Keywords: Electronic apex locator, Advanced diagnostic methods.

\section{INTRODUCTION}

In endodontic treatment for permanent teeth, it is important to estimate the working length precisely. Currently, various methods are used in clinical practice for working length estimation ranges from conventional tactile method to fifth generation apex locators. Considering the safety and accuracy of the procedure, a single method alone may not be enough to satisfy the clinical need. The purpose of this study is to compare the accuracy of root canal length in permanent teeth determined by electronic apex locators, conventional and digital radiography.

\section{The Concept of 'Working Length' in Endodontic Treatment}

Grove, in 1930, stated that 'the proper point to which root canals should be filled is the junction of the dentin and the cementum and that the pulp should be severed at the point of its union with the periodontal membrane'. ${ }^{1}$ The cementodentinal junction
(CDJ) is the anatomical and histological landmark where the periodontal ligament begins and the pulp ends. Root canal preparation techniques aim to make use of this potential natural barrier between the contents of the canal and the apical tissues. It is generally agreed that the preparation and obturation of the root canal should be at or short of the apical constriction. Clinicians often found it difficult to identify and prepare this landmark accurately. Studies have reported that the best prognosis is when the root filling lies within $2 \mathrm{~mm}$ of the radiographic apex. ${ }^{2,3}$ The variations in anatomy of tooth apices both by age and tooth type make this task all the more challenging. ${ }^{4}$

In contemporary endodontic practice, various methods are used for working length estimations. Let us briefly describe various methods here.

\section{Tactile Sensation and Working Length Determination}

Tactile sensation, although useful in experienced hands, has many limitations. The anatomical variations in apical constriction 
location, size, tooth type and age make working length assessment unreliable. In some cases, the canal is sclerosed or the constriction has been destroyed by inflammatory resorption. Even among experienced clinicians, only $60 \%$ could locate the apical constriction by using tactile sense. ${ }^{5}$ A simulation study has found substantial variation between subjects, asked to detect a resistance in a root canal model. ${ }^{6}$ Marked intrasubject differences were also observed in the above simulation experiment.

\section{Use of Radiographs in Working Length Determination}

Both conventional and digital radiographs are widely used for working length determination in endodontic procedures. A radiograph provides a two-dimensional image of a threedimensional structure and is technique sensitive in both its exposure and interpretation. The position of the apical foramen, ${ }^{7}$ dense bone and anatomical structures, secondary dentine and cementum deposition ${ }^{8,9}$ are the major limiting factors for the radiographic working length determination. Studies found that using radiographic working length calculations alone led to instrumentation beyond the apical foramen in $56 \%$ of premolars and $33 \%$ of molars. ${ }^{10}$

Though digital radiographs have several advantages over conventional radiographs such as reduced radiation exposure, speed or image acquisition and the possibility of enhancing or editing the image, studies have proven that the clarity of fine endodontic files and periapical lesions was less with phosphorplate digital images than with conventional film. ${ }^{11}$

\section{Apex Locators and Working Length Determination}

Use of the electronic apex locator to determine working length has still not gained enough popularity among endodontists. This may be due to the fact that early devices which suffered from poor accuracy and did not function properly in the presence of common irrigants. Cost of the instruments and exposure to the technology are also factors. A survey in New Zealand found apex locator use favored by younger practitioners, and users reported a reduced number of radiographs taken during root canal treatment of a maxillary molar. ${ }^{12}$ But, in Japan, there is extensive use of apex locators, but large numbers of radiographs are also taken. ${ }^{13}$ Both in vitro and in vivo have confirmed the accuracy of apex locators. ${ }^{14-16}$ But the use of apex locators along with a pre- and postoperative digital radiograph is advised in most of the circumstances. This study aims to compare the accuracy of measurements obtained through these methods in comparison with the actual working length estimated from extracted tooth.

\section{MATERIALS AND METHODS}

A total of 50 single-rooted permanent teeth with mature apices, were extracted for periodontal reasons and obtained from different sources without caries were studied. The teeth were kept in isotonic sodium chloride solution. Access cavities were prepared using round diamond bur 801/014 (Komet, Lemgo, Germany) in a high-speed hand piece and the canals were located with probe. K-files \#8, \#10 and \#15 (Dentsply Maillefer, Ballaigues, Switzerland) were used. Electronic measurements were done next using third generation, DentAport ZX (J Morita MFG. Corp). The teeth was first embedded in alginate with $0.9 \%$ sodium chloride solution which acts as a conducting gel simulating the periodontium. After irrigating with 3\% sodium hypochlorite, the canals were dried with a paper point. The measurements were carried out in mode 1 (M1, 1 mm short of the apex). Endo gauge was used for measurements.

The radiographic measurements were made using paralleling technique. The distance between the tooth and source, tooth and film and the exposure factors was standardized. Photostimulated phosphor (PSP) was used to take digital radiographs. The sensors were digitized by DentOptix laser light scanner. The images were stored and displayed on screen. By use of computer scanner, working lengths were estimated. Finally, the actual canal length of each tooth was measured using direct observation of the apical exit of the same file. Two readings were taken for each tooth, by the same operator, using the same metallic ruler.

The data was analyzed in STATA 12.0 software. Mean difference with standard deviation and interclass correlation coefficients and failure rate were calculated.

\section{RESULTS}

Mean working length and standard deviations for each measurement were calculated. The mean difference was not statistically significant for electronic apex locators and digital radiographic technique. But in the case of conventional radiography, there is a statistically significant mean difference in the measurements which was obtained while performing the paired t-test.

High interclass correlation coefficients were observed between the apex locator and actual length $[r=0.995$, 95\% CI (0.991-0.997) $\mathrm{p}<0.01$ ], between conventional radiography and actual length [ $\mathrm{r}=0.981,95 \%$ CI $(0.967-0.989) \mathrm{p}<0.01]$, and between digital radiography and actual length $[r=0.980,95 \%$ CI (0.964-0.988) $\mathrm{p}<0.01]$. The measurements obtained through all the three techniques showed excellent level of agreement too. The working length estimated by each method was compared with the actual length (control). The difference between the two measurements was used to calculate the failure rate. Using electronic apex locator, the failure rate that was obtained was $22 \%$ and all of working lengths were shorter than the actual working lengths. The differences between the results from electronic apex locators and control were more than $0.5 \mathrm{~mm}$.

Conventional radiography gave the failure rate of $38 \%$. Half of the failed results were longer and half of them were shorter than control's results. All the failed results were showing the difference of more than $0.5 \mathrm{~mm}$. 


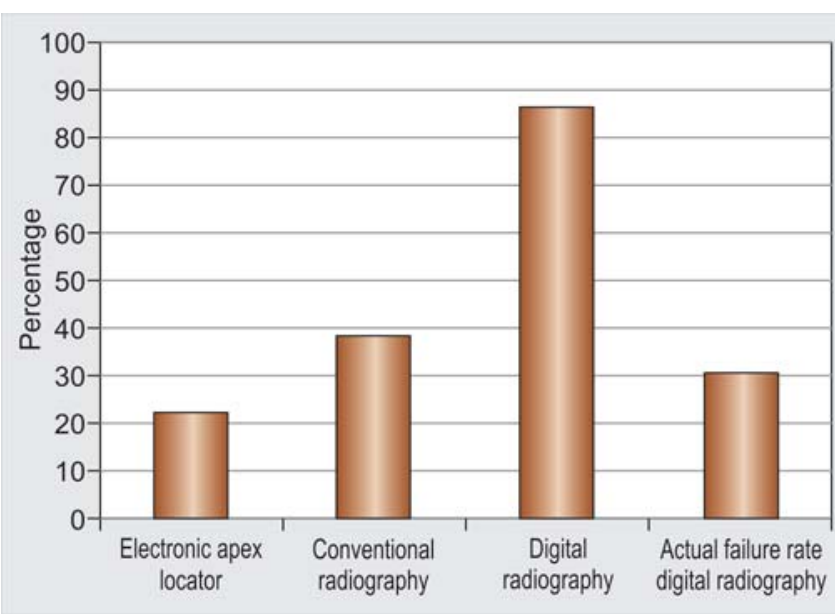

Graph 1: Results of single technique (failure rate)

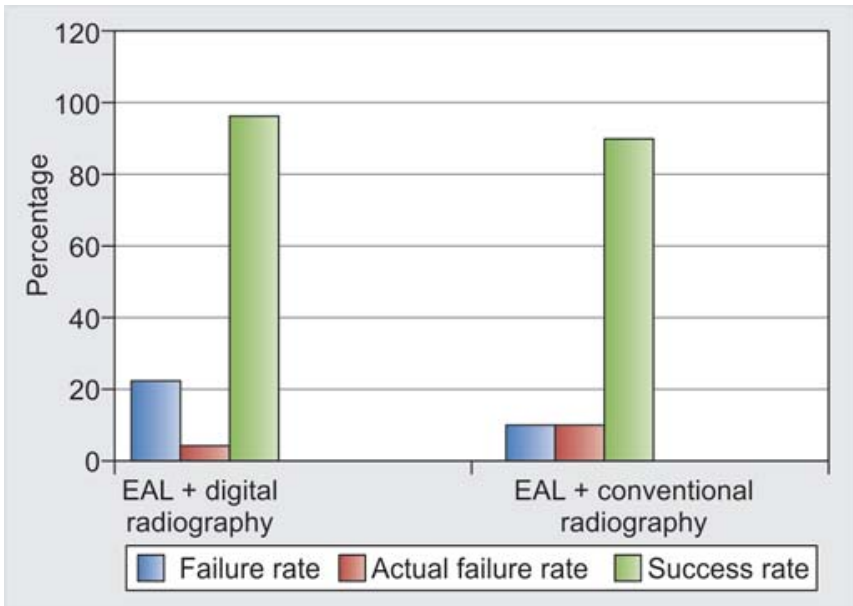

Graph 2: Results of combination technique

Digital radiography resulted in $86 \%$ failure rate but only $30 \%$ were yielded with difference of more than $0.5 \mathrm{~mm}$ from actual working lengths. A total of $56 \%$ resulted in difference of less than $0.5 \mathrm{~mm}$, which is not considered to be actual failure. So, the actual failure rate for digital radiography is considered to be only 30\% (Graph 1).

Further, measurements of two methods were combined and compared it with actual tooth length. From combination technique of electronic apex locator and digital radiography, the failure rate obtained was $22 \%$ but only $4 \%$ had a difference of more than $0.5 \mathrm{~mm}$. Combination of electronic apex locator and conventional radiography was yielded in failure rate of $10 \%$ and all were shown the difference of more than $0.5 \mathrm{~mm}$. So, the success rate of electronic apex locator and conventional radiography is $90 \%$ and the success rate of electronic apex locator and digital radiography is $96 \%$ (Graph 2).

\section{DISCUSSION}

Many studies have confirmed the reliability of electronic apex locators in working length measurement. Using conventional radiographs results in repeated exposure to unwanted dosage of radiations. Studies in animal and human beings have proved the adverse effects of these radiations on oral and maxillofacial regions. ${ }^{17}$ Although digital radiographs were able to overcome many of the disadvantages of conventional radiography, the measurements were not accurate compared to the electronic apex locators. In this study, high-reliability coefficients were observed in all techniques. The failure rate estimates show that apex locators and digital radiographic measurements were best related to actual canal length. These results confirmed previous studies that demonstrate that electronic apex locators can accurately determine the canal length to within $0.5 \mathrm{~mm}$ of the apical foramen. ${ }^{14,15,18,19}$ But the accuracy of apex locators can be affected by different types of electrolytes, foramen size and pulp vitality. This study found the least magnitude of deviation from the mean in measurements by the apex locator. The proponents of apex locators argue that new generations of frequency-dependent apex locators, based on electrical principles, can detect the narrowest diameter of the canal even in the presence of moisture and conductive fluids.

The use of apex locators alone, without a preoperative and postoperative radiograph, is not a recommended practice due to the large variation in tooth morphology. Studies have found that the use of the Root $\mathrm{ZX}$ decreased the overestimation of working length of the premolar group to $2 \% .{ }^{10}$ It is generally agreed that preoperative radiograph is essential in endodontics to determine the anatomy of the root canal system, the number and curvature of roots, the presence or absence of disease and to act as an initial guide for working length. The electronic apex locators, which used with appropriate radiographs, allow for much greater accuracy of working length. ${ }^{16,20}$

\section{CONCLUSION}

From the result of this in vitro study, it can be concluded that a combination of digital radiographic and apex locator methods for determining the root canal length in permanent teeth can be considered safe, reliable and precise.

\section{REFERENCES}

1. Grove C. Why canals should be filled to the dentinocemental junction. J Am Dent Assoc 1930;17:293-96.

2. Dummer PM, McGinn JH, DG R. The position and topography of the apical canal constriction and apical foramen. Int Endod J 1984;17(4):8.

3. Stein TJ, JF C. Anatomy of the root apex and its histologic changes with age. Oral Surg Oral Med Oral Pathol 1990; 69(2):238-42.

4. Chikvashvili J. Electronic apex locators: Determining proper canal length. Parkwell online learning centre.

5. Seidberg BH, Alibrandi BV, Fine H, BL. Clinical investigation of measuring working length of root canals with an electronic device and with digital-tactile sense. J Am Dent Assoc 379-87.

6. Chandler NP, GP B. Effect of gloves on tactile discrimination using an endodontic model. International Endodontic Journal 1990;23:9

7. Oslon AK, Goering AC, Cavataio RE, JL. The ability of the radiograph to determine the location of the apical foramen. International Endodontic Journal 1991;24(1):28-35. 
8. Stein TJ, JF C. Anatomy of the root apex and its histologic changes with age. Oral Surgery, Oral Medicine, Oral Pathology 1990;69:238-42.

9. Chong BS, PFT R. Apex locators in endodontics: Which, when and how? Dental Update 1994;27:328-30.

10. ElAyouti A, RW. Frequency of overinstrumentation with an acceptable radiographic working length. Journal of Endodontics 2001;27:49-52.

11. Shearer AC, Horner K, NH W. Radiovisiography for length estimation in root canal treatment: An in vitro comparison with conventional radiography. Int Endod J 1991;24:233-39.

12. Chandler NP, SK. Radiographic practices of dentists undertaking endodontics in New Zealand. Dentomaxillofacial Radiology 2002;31:217-321.

13. Yoshikawa G, Sawada N, Wettasinghe KA HS. Survey of endodontic treatment in Japan (abstract). Journal of Endodontics 2001;27:236.

14. Dunlap CA, Remeikis NA, BeGole EA, R RC. An in vivo evaluation of an electronic apex locator that uses the ratio method in vital and necrotic canals. Journal of Endodontics 1998;24: 48-50.

15. Ounsi HF, AN. In vitro evaluation of the reliability of the root ZX electronic apex locator. 32 1999:120-23.

16. Pratten DH, JMN. Comparison of radiographic and electronic working lengths. Journal of Endodontics 1996;22:173-76.

17. Yousef saad A, Al-Nazhan. Radiation dose reduction during endodontic therapy: A new technique in combining apex locator and a digital imaging system. Journal of Endodontics 2000;26:144-47.

18. McGinty DT, Fabre DD, Miller DA, LE P, Endodon J. Do irrigants affect the precision of apex locators? Journal of Endodontics 1996;22:195.

19. Schweiz MZ. In vitro comparison of electronic root canal length measuring device and the radiographic determination of working length 2001;111:1165-70.

20. Segura-Egea JJ, Jimenez-Pinzon A, VR SJ. Endodontic therapy in a 3-rooted mandibular first molar: Importance of a thorough radiographic examination. Journal of the Canadian Dental Association 2002;68:541-44. 